\title{
Bounded Synchronization of A Heterogeneous Complex Switched Network ${ }^{\star}$
}

\author{
Lei Wang ${ }^{\mathrm{a}}$, Michael Z. Q. Chen ${ }^{\mathrm{b}}$, Qing-Guo Wang ${ }^{\mathrm{c}}$ \\ ${ }^{a}$ School of Automation Science 8 Electrical Engineering, Beihang University, Beijing, 100191, P. R. China \\ ${ }^{\mathrm{b}}$ Department of Mechanical Engineering, The University of Hong Kong, Hong Kong \\ ${ }^{\mathrm{c}}$ Department of Electrical and Computer Engineering, National University of Singapore, Singapore, 119260
}

\begin{abstract}
This paper investigates synchronization issues of a heterogeneous complex network with a general switching topology in the sense of boundedness, when no complete synchronization manifold exists. Several sufficient conditions are established with the Lyapunov method and the differential analysis of convergence to determine the existence and estimate the convergence domain for the local and global bounded synchronization of a heterogeneous complex network. By using the consensus convergence of a switched linear system associated with the switching topology, explicit bounds of the maximum deviation between nodes are obtained in the form of a scalar inequality involving the property of the consensus convergence, the homogeneous and heterogeneous dynamics of individual nodes for the local and global cases. These analytical results are simple yet generic, which can be used to explore synchronization issues of various complex networks. Finally, a numerical simulation illustrates their effectiveness.
\end{abstract}

Key words: Dynamical networks; heterogeneity; synchronization; switching topology.

\section{Introduction}

Synchronization of populations of locally interacting units is an active field of research with applications in science and engineering, see [Pikovsky et al., 2001], [Osipov, Kurths, \& Zhou, 2007], [Arenas et al., 2008] and references therein. From the viewpoint of complex network, synchronization of complex systems is usually determined by the dynamics of individual nodes and the coupling configuration between nodes. This result has been established by mainly assuming that all the node dynamics are identical, see [Wu, 2005], [Belykh et al., 2006], [Yu et al., 2009], [Stilwell et al. 200)], [Zhao et al. 2009]. However, significant differences commonly exist within the relevant individual nodes. Motivated by this, we investigate synchronization issues of complex heterogeneous networks in this paper.

\footnotetext{
* This paper was not presented at any IFAC meeting. Corresponding author M. Z. Q. Chen. Tel. (852)28592628. Fax (852)28585415.

Email addresses: 1wang@buaa.edu.cn (Lei Wang), mzqchen@hku.hk (Michael Z. Q. Chen), elewqg@nus.edu.sg (Qing-Guo Wang).
}

The behavior of complex dynamical networks with nonidentical nodes is much more complicated than that of the identical-node case since synchronization manifold, guaranteed by the diffusive condition in identical-node networks, disappears due to the heterogeneity of individual nodes. And the ultimate synchronous trajectory, in general, has to be confined to some particular solution. Also, decompositions into a few of lower dimensional subsystems are no longer possible even for local synchronization of heterogeneous networks. Thus, it is quite difficult to explore synchronization of complex heterogeneous networks, and very few results have been reported to date. A simple case for all nonidentical nodes shared with a common equilibrium has been studied in [Xiang \& Chen, 2007], [Zhao et al., 2011]. Besides, synchronization of coupled nonidentical chaotic systems have also been discussed in [Femat et al., 2005], [Li, Chen, \& Aihara, 2006], [Duan \& Chen, 2009].

There is no doubt that a complex network of coupled nonidentical systems may still exhibit some kind of synchronous behaviors that need to be understood. Bounded synchronization is a typical weaker form of synchronization when complete synchronization is impossible. Examples include clock synchronization in mobile robot- 
s, or task coordination of swarming animals, or the appearance of synchronized bulk oscillations in suspension of yeast cells etc. Several investigations have discussed the bounded synchronization issues, e.g., in stochastic complex networks [Shen et al., 2011] and consensus control of multi-agent systems [Zhong et al., 2012]. A very recent result in [Zhao et al., 2012] has addressed synchronization of a general dynamical network with nonidentical nodes and symmetric coupling matrix. Wang et al. [Wang et al., 2015] introduced a generalized connection graph stability method to avoid the calculating eigenvalues of an asymmetry coupling matrix. It is noted that the above mentioned topology has dealt with a "slow-varying" structure, i.e., a continuous function is simply taken as the varying connection for a time-varying network. Such a description has limitations in handling the varying topologies that change very quickly such as switches due to connection failures and new creations. In general, the relevant time-varying results exhibit strong conservativeness, particularly in analyzing an unconnected topology. A few recent results [Frasca et al., 2008], [Wang et al., 2010] have discussed switching synchronization issues of a mobile agent network under the fast-switching constraint. More results on switching networks have focused on the consensus of multi-agent systems from the viewpoint of control science, see [Olfati-Saber \& Murray, 2004], [(Ren \& Beard, 2005)] for example. This paper investigates bounded synchronization of a a heterogeneous complex switched network. With the Lyapunov function approach as well as differential analysis of convergence, we derive several bounded synchronization conditions for the heterogeneous network. In particular, the problem of bounded synchronization under the general switching topology is solved by partially using the techniques of the consensus problem because of the similarity of synchronization in complex coupled networks and consensus in multi-agent systems.

The rest of this paper is organized as follows. Section 2 presents a heterogeneous complex switched network and some mathematical preliminaries. In Section 3, we derive several sufficient conditions to guarantee the local and global bounded synchronization of the considered network, respectively. A numerical example is given to elucidate the effectiveness of the presented results in Section 4 . Section 5 concludes the investigation.

\section{A Heterogeneous Network Model}

Consider a nonlinear system of $N$ linearly and diffusively coupled nonidentical nodes which are represented by

$\dot{\mathbf{x}}_{i}(t)=\mathbf{f}_{i}\left(t, \mathbf{x}_{i}\right)-c \sum_{j=1}^{N} L_{\sigma i j} \Gamma \mathbf{x}_{j}(t), i=1,2, \ldots, N$ where $\mathbf{x}_{i}=\left[x_{i 1}, x_{i 2}, \ldots, x_{i n}\right]^{\mathrm{T}} \in \mathbb{R}^{n}$ is the state vector of node $i, \mathbf{f}_{i}:[0, \infty) \times D \rightarrow \mathbb{R}^{n}$ is continuously differentiable with $D \subseteq \mathbb{R}^{n}$, governing the dynamics of each node, $c>0$ is the overall coupling strength, $\Gamma=$ $\operatorname{diag}\left(\gamma_{1}, \ldots, \gamma_{n}\right) \in \mathbb{R}^{n \times n}$ is the inner-coupling matrix, the switching signal $\sigma(t):[0, \infty) \rightarrow \mathcal{P}=\{1,2, \ldots, p\}$ with $p<\infty$ is a piecewise constant function with successive times to describe the topology switches between subintervals. In network (1), the communication topology is represented by digraph $\mathcal{G}^{\sigma}$ and described in a matrix form by the Laplacian $L_{\sigma(t)}=\left(L_{\sigma i j}\right) \in \mathbb{R}^{N \times N}$. The Laplacian of digraph $\mathcal{G}^{\sigma}$ is defined as follows: If there is a directed connection from node $j$ to node $i(i \neq j)$ at time $t$, then $L_{\sigma i j}<0$; otherwise $L_{\sigma i j}=0$, and its diagonal entries satisfy $L_{\sigma i i}=-\sum_{j=1, j \neq i}^{N} L_{\sigma i j}, \forall i$, and $\sigma(t)$.

For simplicity, let $\mathcal{G}=\left\{\mathcal{G}^{i} \mid i \in \mathcal{P}\right\}$ denote the set of all possible communication graphs of network (1) in the process of switching, each of which represents a digraph with the Laplacian $L_{i}$ for $i \in \mathcal{P}$. Also, consider an infinite sequence of nonempty, bounded and contiguous time intervals $\left[t_{k}, t_{k+1}\right), k=0,1, \ldots$, with $t_{0}=0$ and $t_{k+1}-t_{k} \leq$ $T_{\max }$ for some positive constant $T_{\max }$. In each interval $\left[t_{k}, t_{k+1}\right)$, there is a sequence of non-overlapping subintervals $\left[t_{k_{0}}, t_{k_{1}}\right),\left[t_{k_{1}}, t_{k_{2}}\right), \ldots,\left[t_{k_{m_{k-1}}}, t_{k_{m_{k}}}\right)$ with $t_{k_{0}}=$ $t_{k}, t_{k_{m_{k}}}=t_{k+1}$ satisfying $t_{k_{j+1}}-t_{k_{j}} \geq T_{\min }, 0 \leq j \leq$ $m-1$, for some integer $m \geq 1$ and a given positive constant $T_{\min }$. In particular, the digraph $\mathcal{G}^{\sigma}$ with the Laplacian $L_{\sigma}$ switches at $t_{k_{l}}$ and it does not change during each subinterval $\left[t_{k_{l}}, t_{k_{l+1}}\right)$. Throughout this paper, notations for graphs and their corresponding Laplacian matrices are not differentiated unless stated otherwise.

Assumption 1 (A1): The dynamics of each isolated node can be expressed in the form of $\mathbf{f}_{i}\left(t, \mathbf{x}_{i}\right)=$ $\mathbf{f}\left(t, \mathbf{x}_{i}, \mathbf{X}\right)+\mathbf{g}_{i}(t, \mathbf{X})$ and $\left\|\mathbf{g}_{i}\right\| \leq \delta$ holds uniformly for all nodes with constant $\delta$ as the heterogeneity parameter, where f : $[0, \infty) \times D \times \ldots \times D \rightarrow \mathbb{R}^{n}$, $\mathbf{g}_{i}:[0, \infty) \times D \times \ldots \times D \rightarrow \mathbb{R}^{n}, \mathbf{X}=\left[\mathbf{x}_{1}^{\mathrm{T}}, \mathbf{x}_{2}^{\mathrm{T}}, \ldots, \mathbf{x}_{N}^{\mathrm{T}}\right]^{\mathrm{T}} \in$ $\mathbb{R}^{n N}$, and $\|\cdot\|$ denotes the Euclidean norm.

Note that the heterogeneous dynamics $\mathbf{g}_{i}(t, \mathbf{X})$ represents the differences arising from the individual nodal dynamics. A common choice of $\mathbf{f}$ is $\mathbf{f}\left(t, \mathbf{x}_{i}, \mathbf{X}\right)=$ $\sum_{i=1}^{N} \xi_{i} \mathbf{f}_{i}\left(t, \mathbf{x}_{i}\right)$, where $\xi_{i} \geq 0$ for all $i$ and $\sum_{i=1}^{N} \xi_{i}=1$. Sometimes, one can simply select $\mathbf{f}\left(t, \mathbf{x}_{i}, \mathbf{X}\right) \stackrel{i=1}{=} \mathbf{f}\left(t, \mathbf{x}_{i}\right)$ according to the nodal dynamics for some $i$. Then, $\mathbf{g}_{i}(t, \mathbf{X})=\mathbf{f}_{i}\left(t, \mathbf{x}_{i}\right)-\mathbf{f}\left(t, \mathbf{x}_{i}\right)$, and the estimation $\delta$ associates with the states of nodes. It is noted that $\delta$ can be analytically calculated for many coupled limit-cycle or chaotic systems as if the bound of nodal states is known as a prior. Besides, the function $\mathbf{g}_{i}(t, \mathbf{X})$ can also take the noise or external disturbances into account.

Now, let $\mathbf{F}(t, \mathbf{X})=\left[\mathbf{f}^{\mathrm{T}}\left(t, \mathbf{x}_{1}, \mathbf{X}\right), \ldots, \mathbf{f}^{\mathrm{T}}\left(t, \mathbf{x}_{N}, \mathbf{X}\right)\right]^{\mathrm{T}} \in$ $\mathbb{R}^{n N}, \mathbf{G}(t)=\left[\mathbf{g}_{1}^{\mathrm{T}}(t, \mathbf{X}), \ldots, \mathbf{g}_{N}^{\mathrm{T}}(t, \mathbf{X})\right]^{\mathrm{T}} \in \mathbb{R}^{n N}$. Then, 
network (1) can be rewritten in a block form as

$$
\dot{\mathbf{X}}(t)=\mathbf{F}(t, \mathbf{X})-c\left(L_{\sigma} \otimes \Gamma\right) \mathbf{X}(t)+\mathbf{G}(t),
$$

where $\otimes$ is the Kronecker product. From A1, $\mathbf{G}(t) \equiv 0$ means that the heterogeneity among the node dynamics disappears. Then, network (2) reduces to a complex network of coupled identical nodes

$$
\dot{\mathbf{X}}(t)=\mathbf{F}(t, \mathbf{X})-c\left(L_{\sigma} \otimes \Gamma\right) \mathbf{X}(t)
$$

For network (3), there always exists an invariant synchronization manifold $\mathbf{S}=\left\{\left(\mathbf{x}_{1}^{\mathrm{T}}, \ldots, \mathbf{x}_{N}^{\mathrm{T}}\right)^{\mathrm{T}} \in \mathbb{R}^{n N}\right.$ : $\left.\mathbf{x}_{i}=\mathbf{x}_{j}, \forall i, j\right\}$. We denote an orthonormal basis of $\mathbf{S}$ by $\tilde{\mathbf{v}} \triangleq\left(\mathbf{v}^{\mathrm{T}} \otimes I_{n}\right) \in \mathbb{R}^{n \times n N}$ and a basis of orthocomplement space of $\mathbf{S}$ (denoted by $\left.\mathbf{S}^{+}\right)$by $\tilde{\mathbf{v}}_{+} \triangleq\left(\mathbf{v}_{+}^{\mathrm{T}} \otimes I_{n}\right) \in$ $\mathbb{R}^{n(N-1) \times n N}$, where $\mathbf{v}=\frac{1}{\sqrt{N}}[1,1, \ldots, 1]^{\mathbf{T}} \in \mathbb{R}^{N}$, and $\mathbf{v}_{+} \in \mathbb{R}^{N \times(N-1)}$. Then, it is easy to verify that $\mathbf{X} \in$ $\mathbf{S} \Longleftrightarrow \tilde{\mathbf{v}}_{+} \mathbf{X}=0$. Left-multiplying Eq. (2) by $\tilde{\mathbf{v}}_{+}$yields

$$
\begin{aligned}
\dot{\mathbf{Y}}(t)=\tilde{\mathbf{v}}_{+} \mathbf{F}\left(t, \tilde{\mathbf{v}}_{+}^{\mathrm{T}} \mathbf{Y}(t)+\overline{\mathbf{X}}(t)\right) & \\
& -c\left(\tilde{L}_{\sigma} \otimes \Gamma\right) \mathbf{Y}(t)+\tilde{\mathbf{v}}_{+} \mathbf{G}(t),
\end{aligned}
$$

where $I_{n}$ is an $n \times n$ identity matrix, $\mathbf{Y}=\tilde{\mathbf{v}}_{+} \mathbf{X} \in$ $\mathbb{R}^{n(N-1)}, \overline{\mathbf{X}}=\left[\overline{\mathbf{x}}^{\mathrm{T}}, \overline{\mathbf{x}}^{\mathrm{T}}, \ldots, \overline{\mathbf{x}}^{\mathrm{T}}\right]^{\mathrm{T}} \in \mathbb{R}^{n N}, \overline{\mathbf{x}}=\frac{1}{\sqrt{N}} \tilde{\mathbf{v}} \mathbf{X}$ is the average state trajectory, and $\tilde{L}_{\sigma}=\mathbf{v}_{+}^{\mathrm{T}} L_{\sigma} \mathbf{v}_{+}$. Obviously, $\mathbf{Y}(t)=0$ is an equilibrium point of system

$$
\dot{\mathbf{Y}}(t)=\tilde{\mathbf{v}}_{+} \mathbf{F}(t, \tilde{\mathbf{v}} \mathbf{Y}(t)+\overline{\mathbf{X}}(t))-c\left(\tilde{L}_{\sigma} \otimes \Gamma\right) \mathbf{Y}(t)
$$

The exponential stability of system (5) is equivalen$\mathrm{t}$ to the exponential synchronization of network (3) [Wang \& Wang, 2013]. Correspondingly, the bounded synchronization of network (2) can be assessed by the ultimate boundedness of the solution $\mathbf{Y}(t)$ of system (4).

Definition 1. Network (1) is said to achieve bounded synchronization to the convergence domian $\mathcal{M}$ if $\forall i, j=1, \ldots, N, \mathbf{X}_{i j}(t)$ approaches to $\mathcal{M}$, i.e., $\lim _{t \rightarrow \infty} \operatorname{dist}\left(\mathbf{X}_{i j}(t), \mathcal{M}\right)=0$, where $\mathbf{X}_{i j}(t)=\mathbf{x}_{i}(t)-$ $\mathbf{x}_{j}(t), \operatorname{dist}\left(\mathbf{x}^{*}, \mathcal{M}\right)$ denotes the distance from a point $\mathbf{x}^{*}$ to a set $\mathcal{M}$, that is, the smallest distance from $\mathbf{x}^{*}$ to any point in $\mathcal{M}$.

\section{Bounded Synchronization Analysis}

\subsection{Analysis of local bounded synchronization}

Theorem 1. Suppose that A1 holds. If there exist a uniformly symmetric positive definite matrix $P(t) \in$ $\mathbb{R}^{n(N-1) \times n(N-1)}$ and positive scalars $a, b$, and $\eta$ such that

$$
\begin{gathered}
a\|\mathbf{Y}(t)\|^{2} \leq \mathbf{Y}^{\mathrm{T}}(t) P(t) \mathbf{Y}(t) \leq b\|\mathbf{Y}(t)\|^{2}, \forall \mathbf{Y}(t) \\
\dot{P}(t)+A^{\mathrm{T}}(t) P(t)+P(t) A(t)+2 \eta I_{n(N-1)} \leq 0
\end{gathered}
$$

then network (1) with the initial condition $\mathbf{X}_{i j}\left(t_{0}\right) \in$ $B_{\bar{\delta}}=\left\{\mathbf{Y} \in \mathbb{R}^{n(N-1)} \mid\|\mathbf{Y}\| \leq \bar{\delta}\right\}$ for any $i$ and $j$ synchronizes to the set $\mathcal{M}_{1}=\left\{\mathbf{X} \in \mathbb{R}^{n N} \mid\|\mathbf{X}-\overline{\mathbf{X}}\| \leq\right.$ $\underline{\delta} \sqrt{a / b}\}$, where $A(t)=I_{N-1} \otimes J_{f}(t, \overline{\mathbf{X}})-c \tilde{L}_{\sigma} \otimes \Gamma$, $J_{f}(t, \overline{\mathbf{X}})$ is the Jacobian matrix of $\mathbf{f}(t, \mathbf{X}(t))$ evaluated at $\overline{\mathbf{X}}(t), \phi=\sqrt{\sum_{i=1}^{n} \phi_{i}^{2}}$ with the Hessian matrix $\Phi_{j}$ of function $f_{j}$ satisfying $\left\|\Phi_{j}\right\| \leq \phi_{j}$ for all $j, \underline{\delta}=4 \sqrt{N} \delta /\left(\eta+\sqrt{\eta^{2}-8 \sqrt{n N} \phi \delta}\right)$ and $\bar{\delta}=4 \sqrt{N} \delta /\left(\eta-\sqrt{\eta^{2}-8 \sqrt{n N} \phi \delta}\right)$.

Proof. Use the Taylor formula with the integral remainder at $\overline{\mathbf{x}}$ and write it in a compact form as $\mathbf{F}(t, \mathbf{X})=$ $\sqrt{N} \mathbf{v} \otimes \mathbf{f}(t, \overline{\mathbf{X}})+\left(I_{N} \otimes J_{f}(t, \overline{\mathbf{X}})\right)(\mathbf{X}(t)-\overline{\mathbf{X}}(t))+\mathbf{H}(t)$, where $\mathbf{h}_{i}(t)=\left[h_{i 1}(t), \ldots, h_{i n}(t)\right]^{\mathrm{T}} \in \mathbb{R}^{n}$ with $h_{i j}(t)=$ $\int_{0}^{1}(1-s)\left(\mathbf{x}_{i}-\overline{\mathbf{x}}\right)^{\mathrm{T}} \Phi_{j}\left((1-s) \mathbf{x}_{i}+s \overline{\mathbf{x}}\right)\left(\mathbf{x}_{i}-\overline{\mathbf{x}}\right) d s, \mathbf{H}(t)=$ $\left[\mathbf{h}_{1}^{\mathrm{T}}(t), \ldots, \mathbf{h}_{N}^{\mathrm{T}}(t)\right]^{\mathrm{T}}$. Then, system (4) can be further rewritten as

$$
\dot{\mathbf{Y}}(t)=A(t) \mathbf{Y}(t)+\tilde{\mathbf{v}}_{+}(\mathbf{H}(t)+\mathbf{G}(t)) .
$$

We use $V(t, \mathbf{Y}(t))=\frac{1}{2} \mathbf{Y}^{\mathrm{T}}(t) P(t) \mathbf{Y}(t)$ as a Lyapunov function candidate for system $(8)$, where $P(t)$ is solved by Eq. (7). Then, we calculate the derivative of $V(t, \mathbf{Y}(t))$ along the trajectories of system (8) and recall the constraints in Eqs. (6)-(7) as well as $\|\mathbf{H}(t)\| \leq \frac{\sqrt{n}}{2} \phi\|\mathbf{Y}(t)\|^{2}$ to derive

$$
\dot{V}(t) \leq \delta b\|\mathbf{Y}(t)\|-\eta\|\mathbf{Y}(t)\|^{2}+\frac{\sqrt{n}}{2} \phi b\|\mathbf{Y}(t)\|^{3} .
$$

It follows from Eq. (9) that there exists a $K$-class function $\alpha(\|\mathbf{Y}(t)\|)$ such that $\dot{V}(t, \mathbf{Y}(t)) \leq-\alpha(\|\mathbf{Y}(t)\|), \forall \underline{\delta} \leq$ $\|\mathbf{Y}(t)\| \leq \bar{\delta}$. Taking $\epsilon=b \underline{\delta}^{2}$ ensures that $B_{\underline{\delta}} \subset \Omega_{t, \epsilon}=$ $\left\{\mathbf{Y} \in \mathbb{R}^{n(N-1)} \mid V(t, \mathbf{Y}(t)) \leq \epsilon\right\}$. Since $\dot{V}(t, \mathbf{Y}(t)) \leq 0$ on $B_{\bar{\delta}}$, for any $t_{0}$ and any $\overline{\mathbf{Y}}\left(t_{0}\right) \in \Omega_{t_{0}, \epsilon}$, the solution starting at $\left(t_{0}, \mathbf{Y}\left(t_{0}\right)\right)$ stays in $\Omega_{t, \epsilon}$. Therefore, any solution starting in $B_{\bar{\delta}}$ will stay in $\Omega_{t, \epsilon}$ for all future time. To calculate the ultimate bound on $\mathbf{Y}(t)$, we have

$$
V(t, \mathbf{Y}(t)) \leq \epsilon \Longrightarrow a\|\mathbf{Y}(t)\|^{2} \leq \epsilon \Longleftrightarrow\|\mathbf{Y}(t)\| \leq \underline{\delta} \sqrt{a / b} .
$$

Therefore, the ultimate boundedness of system (8) can be taken as $\underline{\delta} \sqrt{a / b}$. The proof is thus completed.

Remark 1. As shown in [Zhao et al., 2012], the linear matrix inequality (7) is a sufficient condition for local exponential synchronization of network (3). Actually, the nonautonomous system (5) can be rewritten as a linear time-varying system by using the Mean Value Theorem

$$
\dot{\mathbf{Y}}(t)=A(t) \mathbf{Y}(t)+\tilde{\mathbf{v}}_{+} \boldsymbol{\Sigma}(t) \tilde{\mathbf{v}}_{+}^{\mathrm{T}} \mathbf{Y}(t),
$$

where $\boldsymbol{\Sigma}(t)=\operatorname{diag}\left(\frac{\partial \mathbf{f}}{\partial \mathbf{x}}\left(t, \mathbf{Z}_{1}\right)-\frac{\partial \mathbf{f}}{\partial \mathbf{x}}(t, \overline{\mathbf{X}}), \ldots, \frac{\partial \mathbf{f}}{\partial \mathbf{x}}\left(t, \mathbf{Z}_{N}\right)-\right.$ $\left.\frac{\partial \mathbf{f}}{\partial \mathbf{x}}(t, \overline{\mathbf{X}})\right) \in \mathbb{R}^{n N \times n N}$, and $\mathbf{Z}_{i}$ is a vector on the line seg- 
ment connecting $\mathbf{X}$ to $\overline{\mathbf{X}}$. Then, according to the converse Lyapunov theorem, if the origin of system (10) is exponentially stable, there always exists a time-varying quadratic Lyapunov function $V(t)$ defined for system (10). Then, the exponential stability of the synchronization manifold in network (3) guarantees the existence of function $V(t)$. Thus, one can easily estimate the boundedness of synchronization from Theorem 1 as if network (3) achieves exponential synchronization.

Remark 2. Consider the solvability of inequality (7). If $P(t)=P_{1} \otimes P_{2}(t)$, then inequality (7) can be guaranteed by the following two inequalities:

$$
\begin{array}{r}
\dot{P}_{2}(t)+J_{f}^{\mathrm{T}}(t, \overline{\mathbf{X}}) P_{2}+P_{2} J_{f}(t, \overline{\mathbf{X}})-\beta_{1} P_{2} \Gamma \leq 0, \\
\beta_{1} P_{1}-c \tilde{L}_{\sigma}^{\mathrm{T}} P_{1}-c P_{1} \tilde{L}_{\sigma}+\beta_{2} I_{N} \leq 0
\end{array}
$$

where constant matrix $P_{1} \in \mathbb{R}^{(N-1) \times(N-1)}$ is positive definite, diagonal matrix $P_{2}(t) \in \mathbb{R}^{n \times n}$ is uniformly positive definite, $\beta_{1}$ and $\beta_{2}$ are arbitrary positive constants. It is noted that Eq. (11) implies the local stability of linear system

$$
\dot{\mathbf{x}}(t)=\left(J_{f}(t, \overline{\mathbf{X}})-\beta_{1} \Gamma\right) \mathbf{x}(t), \forall \mathbf{x}(t) \in \mathbb{R}^{n} .
$$

As shown in the connection graph stability method [Belykh et al., 2004], the existence of $P_{2}$ has been widely investigated when $P_{2}$ is a constant matrix. And the inequality (12) implies the exponential stability of the linear switched system written as

$$
\dot{\mathbf{y}}(t)=-\left(c \tilde{L}_{\sigma}-\beta_{1} I_{N-1}\right) \mathbf{y}(t), \forall \mathbf{y}(t) \in \mathbb{R}^{N-1} .
$$

The following lemma provides a simple convergence domain when the inner-coupling matrix is an identity matrix and its proof is given in Appendix A.

Lemma 1. The solution of the linear switched system

$$
\dot{\tilde{\mathbf{Z}}}(t)=\left(I_{N-1} \otimes J_{f}(t, \overline{\mathbf{x}})-c\left(\tilde{L}_{\sigma} \otimes I_{n}\right)\right) \tilde{\mathbf{Z}}(t)
$$

satisfies $\|\tilde{\mathbf{Z}}(t)\| \leq e^{\left(-\mu+\nu_{\max }+\epsilon\right)\left(t-t_{0}\right)}\left\|\tilde{\mathbf{Z}}\left(t_{0}\right)\right\|$ for a sufficiently small scalar $\epsilon$.

Theorem 2. Suppose that $\mathbf{A} 1$ holds. If $\nu_{\max }-\mu<0$, then network (1) with $\Gamma=I_{n}$ locally synchronizes to the set $\mathcal{M}_{2}=\left\{\mathbf{X} \in \mathbb{R}^{n N} \mid\|\mathbf{X}-\overline{\mathbf{X}}\| \leq \frac{\delta}{\mu-\nu_{\max }}\right\}$, where $\nu_{\max }$ is the largest Lyapunov exponent of system

$$
\dot{\xi}(t)=J_{f}(t, \overline{\mathbf{X}}) \xi(t),
$$

and $\mu$ is the convergence rate of switched system

$$
\dot{\mathbf{w}}(t)=-c \tilde{L}_{\sigma} \mathbf{w}(t) .
$$

Proof. It is well-known that for the local synchronization issue of system (2), we can investigate the linearized system (4) written as

$$
\dot{\mathbf{Z}}=\left(I_{N-1} \otimes J_{f}(t, \overline{\mathbf{X}})-c \tilde{L}_{\sigma} \otimes I_{n}\right) \mathbf{Z}+\tilde{\mathbf{v}}_{+} \mathbf{G},
$$

where the solution of system (18) can be expressed by

$$
\mathbf{Z}(t)=\tilde{\Phi}\left(t, t_{0}\right) \mathbf{Z}(0)+\int_{t_{0}}^{t} \tilde{\Phi}(t, \tau) \tilde{\mathbf{v}}_{+} \mathbf{G}(\tau) d \tau
$$

state transition matrix $\tilde{\Phi}(t, \tau)=\Phi(t) \Phi^{-1}(\tau)$, and $\Phi(t) \in \mathbb{R}^{n \times n}$ is a fundamental solution of system (15).

From the definition of an induced matrix norm, we have $\left\|\tilde{\Phi}\left(t, t_{0}\right)\right\|=\max _{\|\tilde{\mathbf{Z}}\|=1}\left\|\tilde{\Phi}\left(t, t_{0}\right) \tilde{\mathbf{Z}}\right\|$. It follows from Lemma 1 that

$$
\left\|\tilde{\Phi}\left(t, t_{0}\right)\right\| \leq \max _{\|\tilde{\mathbf{Z}}\|=1} e^{\left(-\mu+\nu_{\max }+\epsilon\right)\left(t-t_{0}\right)}\|\tilde{\mathbf{Z}}\| .
$$

Combining it with Eq. (19) and taking the limit give the ultimate boundedness of $\mathbf{Y}(t)$.

Remark 3. Theorem 2 provides a reasonably simple boundedness criterion of local synchronization, relating to the heterogeneity of individual nodes, the largest Lyapunov exponent $\nu_{\max }$ of an isolated homogeneous system (16), and the convergence rate $\mu$ of a linear switched system (17). In particular, if $\delta=0$, then Theorem 2 reduces to a criterion of local synchronization of a linearly coupled complex network. A similar result can also be found in Refs. [Wang \& Wang, 2013,Xiang \& Wei, 2011].

\subsection{Analysis of global bounded synchronization}

Assumption 2 (A2): Suppose that there exist a timevarying matrix $B(t) \in \mathbb{R}^{n \times n}$ and a vector field $\tilde{\mathbf{g}}_{i}(t)$ for $i=1, \ldots, N$ such that $\mathbf{f}_{i}\left(t, \mathbf{x}_{i}\right)=B(t) \mathbf{x}_{i}+\tilde{\mathbf{g}}_{i}(t)$, where $\left\|\tilde{\mathbf{v}}_{+} \tilde{\mathbf{G}}(t)\right\| \leq \tilde{\delta}$ for some positive scalar $\tilde{\delta}$ with $\tilde{\mathbf{G}}(t)=$ $\left[\tilde{\mathbf{g}}_{1}^{\mathrm{T}}(t), \ldots, \overline{\mathbf{g}}_{N}^{\mathrm{T}}(t)\right]^{\mathrm{T}}$, and the fundamental solution of the linear system $\dot{\mathbf{x}}=B(t) \mathbf{x}$, denoted by $\tilde{\Psi}(t)$, satisfying $\|\tilde{\Psi}(t)\| \leq e^{v_{b} t}$ for some constant $v_{b}$.

Theorem 3. Suppose that A2 holds and system (17) achieves global exponential stability, i.e., there exists a positive scalar $\mu$ such that $\|\mathbf{w}(t)\| \leq\left\|\mathbf{w}\left(t_{0}\right)\right\| e^{-\mu t}, \forall \mathbf{w}\left(t_{0}\right) \in$ $\mathbb{R}^{N-1}$. If $\mu>\nu_{b}$, then network (1) globally synchronizes to the set $\mathcal{M}_{3}=\left\{\mathbf{X} \in \mathbb{R}^{n N} \mid\|\mathbf{X}-\overline{\mathbf{X}}\| \leq \frac{\tilde{\delta}}{\mu-\nu_{b}}\right\}$.

The detailed proof is omitted here since it can be easily deduced by following the proof of Theorem 2 .

Remark 4. A2 is quite reasonable since, for a general nonlinear vector function $\mathbf{f}_{i}(t, \mathbf{x})$, it can be easily decomposed into a linear part $B \mathbf{x}$ with constant matrix $B$ and 
a nonlinear part $\tilde{\mathbf{g}}_{i}(t)$. Then, $\mathbf{A} \mathbf{2}$ is consistent with $\mathbf{A} \mathbf{1}$. However, such a simple decomposition is sometimes not a good choice to obtain smaller scalars $\nu_{b}$ and $\tilde{\delta}$. By using the Taylor formula, we may decompose $\mathbf{f}_{i}\left(t, \mathbf{x}_{i}\right)$ into

$$
\mathbf{f}_{i}\left(t, \mathbf{x}_{i}\right)=\mathbf{f}(t, \overline{\mathbf{X}})+J_{f}(t, \overline{\mathbf{X}})\left(\mathbf{x}_{i}-\overline{\mathbf{x}}\right)+\mathbf{g}_{i}(t)+\mathbf{r}\left(t, \mathbf{x}_{i}\right),
$$

where $\mathbf{r}\left(t, \mathbf{x}_{i}\right) \in \mathbb{R}^{n}$ is the integral form of the reminder, $B(t)=J_{f}(t, \overline{\mathbf{X}})$, and $\tilde{\mathbf{g}}_{i}=\mathbf{f}(t, \overline{\mathbf{X}})-J_{f}(t, \overline{\mathbf{X}}) \overline{\mathbf{x}}+\mathbf{g}_{i}(t)+$ $\mathbf{r}\left(t, \mathbf{x}_{i}\right)$. Then a conservative condition that guarantees $\mathbf{A 2}$ can be given as $\left.\tilde{\delta} \triangleq \delta+\| \mathbf{R}\left(t, \mathbf{x}_{i}\right)\right) \|$.

Remark 5. Theorem 2 and Theorem 3 require the convergence rate $\mu$. For system (17), the convergence rate $\mu$ is equal to the consensus convergence rate of multi-agent system written as

$$
\dot{\mathbf{x}}_{i}(t)=-c \sum_{j=1}^{N} L_{\sigma i j} \mathbf{x}_{j}(t), i=1, \ldots, N
$$

A simple estimation of the convergence rate $\mu$ can be calculated as shown in [Xiang \& Wei, 2011].

For a general global synchronization issue of network (1), we here give a relatively conservative result by constructing a quadratic Lyapunov function.

Theorem 4. Suppose that A1 holds. If there exist a uniformly symmetric positive definite matrix $P(t) \in$ $\mathbb{R}^{n(N-1) \times n(N-1)}$, positive scalars $a, b, \alpha$ and $\theta$ such that

$$
\begin{aligned}
& a\|\mathbf{Y}(t)\|^{2} \leq \mathbf{Y}^{\mathrm{T}}(t) P(t) \mathbf{Y}(t) \leq b\|\mathbf{Y}(t)\|^{2}, \forall \mathbf{Y}(t) \\
& \frac{1}{2}(P(t) \Gamma+\Gamma P(t))-\theta P(t) \geq 0 \\
& \mathbf{X}_{i j}^{\mathrm{T}}(t)\left(\frac{1}{2} \dot{P}(t)-\alpha P(t)\right) \mathbf{X}_{i j}(t) \\
& \quad+\left(\mathbf{f}\left(t, \mathbf{x}_{i}, \mathbf{X}\right)-\mathbf{f}\left(t, \mathbf{x}_{j}, \mathbf{X}\right)\right)^{\mathrm{T}} P(t) \mathbf{X}_{i j} \leq 0
\end{aligned}
$$

and there exists an infinite sequence $\left\{t_{k}, k=0,1, \ldots,\right\}$ such that

$$
\left(t_{k+1}-t_{k}\right)^{-1} \int_{t_{k}}^{t_{k+1}} \gamma_{\sigma(t)} d t \geq \gamma
$$

then network (1) globally synchronizes to the set $\mathcal{M}_{4}=\left\{\mathbf{X} \in \mathbb{R}^{n N} \mid\|\mathbf{X}-\overline{\mathbf{X}}\| \leq \frac{b \delta \tilde{\gamma}\left(1-\gamma^{* m+1}\right)}{a\left(1-e^{-\frac{1}{2} \gamma T_{\min }}\right)\left(1-\gamma^{*}\right)}\right\}$, where $\gamma_{\sigma}=c \lambda_{2}^{\sigma} \theta-\alpha, \lambda_{2}^{\sigma}$ is the smallest eigenvalue of matrix $\tilde{L}_{\sigma}$ except zero, $\gamma$ is a positive constant, $\tilde{\gamma}=\max _{\forall l \in \mathbb{N}}\left\{\frac{1}{\gamma_{\sigma}}\left(1-e^{-\frac{1}{2} \gamma_{\sigma}\left(t_{k_{l+1}}-t_{k_{l}}\right.}\right)\right\}$, and $\gamma^{*}=\max _{\forall l \in \mathbb{N}}\left\{e^{-\frac{1}{2} \gamma_{\sigma}\left(t_{k_{l+1}}-t_{k_{l}}\right)}\right\}$.

Proof. Choose the Lyapunov function as

$$
V(t, \mathbf{X}(t))=\frac{1}{2} \mathbf{X}^{\mathrm{T}}(t)(\Pi \otimes P(t)) \mathbf{X}(t),
$$

where $P(t)$ is solved by Eq. (22)-(24). Differentiating $V(t, \mathbf{X}(t))$ along the trajectory of network (2) gives

$$
\begin{aligned}
\dot{V}(t)= & \frac{1}{4 N} \sum_{i, j=1}^{N} \mathbf{X}_{i j}^{\mathrm{T}}(t)\left(\dot{P}(t) \mathbf{X}_{i j}(t)+2 P(t)\left(\mathbf{f}\left(t, \mathbf{x}_{i}, \mathbf{X}\right)\right.\right. \\
& \left.\left.-\mathbf{f}\left(t, \mathbf{x}_{j}, \mathbf{X}\right)\right)\right)+\mathbf{Y}^{\mathrm{T}}(t)\left(I_{N} \otimes P(t)\right) \mathbf{G}(t) \\
& \quad-c \mathbf{Y}^{\mathrm{T}}(t)\left(\hat{L}_{\sigma} \otimes P(t) \Gamma\right) \mathbf{Y}(t) \\
\leq & -\frac{1}{2 N} \sum_{i, j=1}^{N} \gamma_{\sigma} \mathbf{X}_{i j}^{\mathrm{T}}(t) P(t) \mathbf{X}_{i j}(t)+b \delta\|\mathbf{Y}(t)\| .
\end{aligned}
$$

As we know, the Laplacian $L_{\sigma}$ in each time subinterval is constant. Then, in the subinterval $\left[t_{k_{l}}, t_{k_{l+1}}\right)$, the derivative of $V(t)$ satisfies

$$
\dot{V}(t) \leq-\gamma_{\sigma} V(t)+b \delta\|\mathbf{Y}(t)\|
$$

Recalling Eq. (22) and taking $U(t)=\sqrt{V(t)}$, we use $\dot{V}(t)=2 U(t) \dot{U}(t)$ to obtain $\dot{U}(t) \leq-\frac{1}{2} \gamma_{\sigma} U(t)+\frac{b \delta}{2 \sqrt{a}}$. Moreover, by applying the comparison lemma, we have

$$
U\left(t_{k_{l+1}}\right) \leq e^{-\frac{1}{2} \gamma_{\sigma} \Delta t_{k_{l}}} U\left(t_{k_{l}}\right)+\frac{b}{\sqrt{a}} \delta \tilde{\gamma}
$$

where $\Delta t_{k_{l}}=t_{k_{l+1}}-t_{k_{l}}$. Note that the common Lyapunov function $V(t)$ is a continuous function in the interval $\left[t_{k}, t_{k+1}\right)$. Then, according to Eq. (28), we deduce a relation by recursion

$$
\begin{aligned}
U\left(t_{k}\right) \leq e^{-\frac{1}{2} \int_{0}^{t_{k}} \gamma_{\sigma} d t} U(0) & \\
& +\frac{b \delta \tilde{\gamma}\left(1-e^{-\frac{1}{2} \gamma T_{\min }(k+1)}\right)\left(1-\gamma^{* m+1}\right)}{\sqrt{a}\left(1-e^{-\frac{1}{2} \gamma T_{\min }}\right)\left(1-\gamma^{*}\right)}
\end{aligned}
$$

Taking the limit on both sides of Eq. (29) indicates that all trajectories $\mathbf{Y}(t)$ converge to the set $\mathcal{M}_{4}$.

Remark 6. The inequality (24) in Theorem 4 implies the exponential stability of the time-varying systems $\forall i, j$,

$$
\dot{\mathbf{X}}_{i j}=\left(\int_{0}^{1} J_{f}\left(t, s \mathbf{x}_{i}+(1-s) \mathbf{x}_{j}\right) d s-\alpha I_{n}\right) \mathbf{X}_{i j}
$$

The detailed proof can be easily deduced by constructing the Lyapunov functions $V_{i j}(t)=\frac{1}{2} \mathbf{X}_{i j}^{\mathrm{T}}(t) Q(t) \mathbf{X}_{i j}(t)$ for each system in Eq. (30). In synchronization analysis and control issues of complex dynamical networks, see [Wang \& Chen, 2002], [Li et al., 2004], [Pham \& Slotine, 2007], [Chen et al. 2007], a sufficiently large $\alpha$ can ensure the existence of $P(t)$. And inequality (22) is solvable by setting $P(t)$ as a constant matrix for the trivial case that all nodes are identical in many coupled limit-cycle or chaotic systems [Belykh et al., 2006]. 

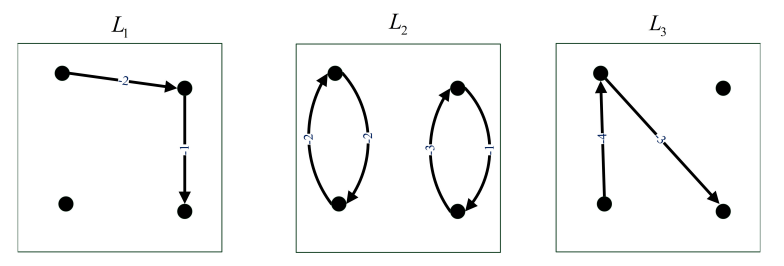

Fig. 1. The switching topologies.

Remark \%. It is observed that from inequality $(25), \bar{\lambda}_{2}^{\sigma} \triangleq$ $\frac{1}{\Delta t_{k}} \int_{t_{k}}^{t_{k+1}} \lambda_{2}^{\sigma} d t$ is the time average of the second smallest eigenvalues corresponding to the Laplacians of switching topology, and $\alpha$ is a constant, which is determined by the dynamics of individual node. As a matter of fact, the inequality (25) indicates the exponential synchronization of the considered switched network (3). A recen$\mathrm{t}$ result can also be derived from Theorem 4 by setting the Laplacians to be symmetric [Wang \& Wang, 2011] or asymmetric [Wang \& Wang, 2012], which indicates that synchronization for a linearly coupled network with a switching topology can be evaluated by the time average of second smallest eigenvalues corresponding to the Laplacians of switching topology.

\section{A Numerical Simulation}

Our simulation model is built on the laboratory devices of coupled pendulums [Fradkov et al., 2005], where each node is a similar pendulum, and every two pendulums are connected by an elastic link. The dynamic equations of the nodes are described by $\dot{x}_{i 1}(t)=x_{i 2}(t)+u_{i 1}(t), \dot{x}_{i 2}(t)=-r_{i} \sin \left(x_{i 1}\right)-$ $q x_{i 2}(t)+\phi_{i}(t)+u_{i 2}(t)$, where $i=1, \ldots, 4, q=3.15$ and $r_{i}=0.02 i$ are system parameters, $\left[\phi_{1}, \phi_{2}, \phi_{3}, \phi_{4}\right]^{\mathrm{T}}=$ $[0.2 \sin t,-0.15 \sin t, 0.1 \sin t,-0.05 \sin t]^{\mathrm{T}}$ are disturbance inputs, $\mathbf{u}_{i}(t)=\left[u_{i 1}(t), u_{i 2}(t)\right]^{\mathrm{T}}$ are control signals that are selected as the distributed consensus protocol: $\mathbf{u}_{i}(t)=-c \sum_{j=1}^{4} L_{i j \sigma} \mathbf{x}_{j}(t)$. The collection of switching topologies is shown in Fig. 1.

Every second, $\sigma(t)$ updates its value in the order of $1,2,3, r, 1,2,3, r, \ldots$, where $r$ is a random valuable randomly chosen from the set $\{1,2,3\}$. For $T=4$, the transition matrix $\Xi_{\sigma}(k T,(k-1) T)$ corresponding to linear system (15) must be one of the three matrices written as $\Xi_{i}=\mathbf{v}_{+}^{\mathrm{T}} e^{-c L_{i}} e^{-c L_{3}} e^{-c L_{2}} e^{-c L_{1}} \mathbf{v}_{+}$with $i=1,2,3$. According to [Xiang \& Wei, 2011], $\mu=2.7784$ by setting the overall coupling strength $c=4$. On the other hand, one can select $B=[0,1 ; 0,-q]$ and $\tilde{\mathbf{g}}_{i}=\left[0,-r_{i} \sin \left(x_{i 1}\right)+\right.$ $\left.\phi_{i}\right]^{\mathrm{T}}$. We then derive $\nu_{b}=0$ and $\tilde{\delta}=0.295$.

Thus, by Theorem 3, it can be concluded that system (1) with such a randomly switching topology achieves global bounded synchronization, and the ultimate bounded regions are $\mathcal{M}_{4}=\{\mathbf{X}\|\mathbf{X}-\overline{\mathbf{X}}\|<0.106\}$. The simulation results are shown in Fig. 2, which shows that the global bounded synchronization of the nonlinear networked system can be guaranteed.
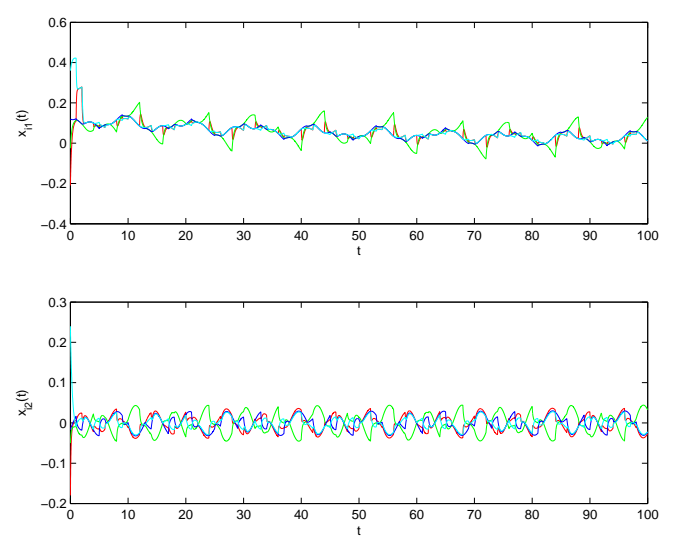

Fig. 2. Trajectories of the complex network.

\section{Conclusions}

Bounded synchronization has been studied for a heterogeneous complex network with a general switching topology in this paper. By using the Lyapunov function approach and the differential analysis of convergence, several convergence domain estimates are derived for the local and global bounded synchronization of a heterogeneous network. In particular, we have shown that the ultimate boundedness of synchronization can be easily evaluated by the consensus convergence rate of a switched linear network associated with the switching topology, the homogeneous and heterogeneous dynamics of individual nodes. Our results are quite simple and powerful, without assumption of a symmetric or connective Laplacian matrix, which can be widely used to investigate various topologies, no matter they are undirected or directed, weighted or unweighted, time-invariant or switching. All these results provide an insight into the analysis and regulation of collective behavior of complex dynamical systems.

\section{Acknowledgements}

This work is supported by the National Natural Science Foundation of China under Grant Nos. 61374053 and 61473016 and HKU Conference and Research Council under Grant 201111159110.

\section{References}

[Pikovsky et al., 2001] Pikovsky, A., Rosenblum, M., \& Kurths, J. (2001). Synchronization, a universal concept in nonlinear sciences, New York: Cambridge University Press.

[Osipov, Kurths, \& Zhou, 2007] Osipov, G. V., Kurths, J., \& Zhou, C. S. (2007). Synchronization in Oscillatory Networks, Berlin: Springer. 
[Arenas et al., 2008] Arenas, A., Díaz-Guilera, A., Kurths, J., Moreno, Y., \& Zhou, C. S. (2008). Synchronization in complex networks, Physcs Reports, 469, 93-153.

[Wu, 2005] Wu, C. W. (2005). Synchronization in networks of nonlinear dynamical systems coupled via a directed graph, Nonlinearity, 18, 1057-1064.

[Belykh et al., 2006] Belykh, I., Belykh, V., \& Hasler, M. (2006). Synchronization in asymmetrically coupled networks with node balance, Chaos, 16, 015102.

[Yu et al., 2009] Yu, W., Cao, J., Chen, G., Lü, J., Han, J., \& Wei, W. (2009). Local synchronization of a complex network model, IEEE Transactions on Systems, Man, and Cybernetics, Part B, 39, 230-241.

[Stilwell et al. 200)] Stilwell, D. J., Bollt, E. M., \& Roberson, D. G. (2006). Sufficient conditions for fast switching synchronization in time varying network topologies, SIAM Journal on Applied Dynamical Systems, 5, 140-156.

[Zhao et al. 2009] Zhao, J., Hill, D. J., \& Liu, T. (2009). Synchronization of complex dynamical networks with switching topology: a switched system point of view, Automatica, 45, 2502-2511.

[Wang \& Wang, 2013] Wang, L., \& Wang, Q. G. (2013). A general appraoch for synchronization of nonlinear networked systems with switching topology, International Journal of Systems Science, 44, 2199-2210.

[Xiang \& Chen, 2007] Xiang, J. \& Chen, G. R. (2007). On the $V$-stability of complex dynamical networks", Automatica, 43, 1049-1057.

[Zhao et al., 2011] Zhao, J., Hill, D. J., \& Liu, T. (2011). Stability of dynamical networks with non-identical nodes: a multiple V-Lyapunov function method, Automatica, 47, 2615-2625.

[Femat et al., 2005] Femat, R., Kocarev, L., van Gerven, L., \& Monsivais-Pérez M. E. (2005). Towards generalized synchronization of strictly different chaotic systems, Physical Letters A, 342, 247-255.

[Li, Chen, \& Aihara, 2006] Li, C., Chen, L., \& Aihara, K. (2006). Synchronization of coupled nonidentical genetic oscillators, Physical Biology, 3, 37-44.

[Duan \& Chen, 2009] Duan, Z. S. \& Chen, G. R. (2009). Global robust stability and synchronization of networks with Lorenztype nodes, IEEE Transactions on Circuits and Systems II, 56(8), 679-683.

[Shen et al., 2011] Shen, B., Wang, Z., \& Liu, X. (2011). Bounded $H_{\infty}$ synchronization and state estimation for discrte timevarying stochastic complex networks over a finite horizon, IEEE Transactions on Neural Networks, 22, 145-157.

[Zhong et al., 2012] Zhong, W. S., Liu, G. P., \& Thomas, C. (2012). Global bounded consensus of multiagent systems with nonidentical nodes and time delays, IEEE Transactions on Systems, Man, and Cybernetics, Part B, 42, 1480-1488.

[Zhao et al., 2012] Zhao, J., Hill, D. J., \& Liu, T. (2012). Global bounded synchronization of general dynamical networks with nonidentical nodes, IEEE Transactions on Automatic Control, 57, 2656-2662.

[Wang et al., 2015] Wang, L., Qian, W., \& Wang Q. G. (2015). Bounded synchronization of a time-varying dynamical network with nonidentical nodes, International Journal of Systems Science, 46, 1234-1245.

[Frasca et al., 2008] Frasca, M., Buscarino, A., Rizzo, A., Fortuna, L., \& Boccaletti, S. (2008). Synchronization of moving chaotic agents, Physacal Review Letters, 100, 044102.
[Wang et al., 2010] Wang, L., Shi, H., \& Sun, Y. X. (2008). Induced synchronization of a mobile agent network by phase locking, Physacal Review E, 82, 046222.

[Olfati-Saber \& Murray, 2004] Olfati-Saber, R. \& Murray, R. M. (2004). Consensus problems in networks of agents with switching topology and time-delays, IEEE Transactions on Automatic Control, 49, 1520-1533.

[(Ren \& Beard, 2005)] Ren, W. \& Beard, R. W. (2005). Consensus seeking in multiagent systems under dynamically changing interaction topologies, IEEE Transactions on Automatic Control, 50, 655-661.

[Belykh et al., 2004] Belykh, V., Belykh, I., \& Hasler, M. (2004). Connection graph stability method for synchronized coupled chaotic systems, Physica D, 195, 159-187.

[Xiang \& Wei, 2011] Xiang, J. \& Wei, W. (2011). On local synchronisability of nonlinear networked systems with a unit inner-coupling matrix and switching topology, International Journal of Control, 84, 1769-1778.

[Wang \& Chen, 2002] Wang, X. F. \& Chen , G. R. (2002). Synchronization in scale-free dynamical networks: robustness and fragility, IEEE Transactions on Circuits and Systems I, $49,54-62$.

[Li et al., 2004] Li, X., Wang, X. F., \& Chen, G. R. (2004). Pinning a complex dynamical network to its equilibrium, IEEE Transactions on Circuits and Systems I, 51, 2074-2087.

[Pham \& Slotine, 2007] Pham, Q. C. \& Slotine, J. J. E. (2007). Stable concurrent synchronization in dynamic system networks, Neural Networks, 20, 62-77.

[Chen et al. 2007] Chen, T. P., Liu, X. W., \& Lu, W. L. (2007). Pinning complex networks by a single controller, IEEE Transactions on Circuits and Systems I, 54, 1317-1326.

[Wang \& Wang, 2011] Wang, L., \& Wang, Q. G. (2011). Synchronization in complex networks with switching topology, it Physics Letters A, 375, 3070-3074.

[Wang \& Wang, 2012] Wang, L., \& Wang, Q. G. (2012). Average contraction and synchronization of complex switched networks, Journal of Physics A: Mathematical and Theoretical, 45, 205101.

[Fradkov et al., 2005] Fradkov, A., Andrievsky, B., \& Boykov, K. (2005). Control of the coupled double pendulums system, Mechatronics, 15, 1289-1303.

[Adrianova, 1995] Adrianova, L. Y. (1995). Introduction to Linear Systems of Differential Equations, New York: American Mathematical Society.

\section{A The proof of Lemma 1}

Proof. Let $\Psi(t)$ be a fundamental solution of system (16), then $\dot{\Psi}(t)=J_{f}(t, \overline{\mathbf{x}}) \Psi(t)$ and $\frac{d}{d t} \Psi^{-1}(t)=$ $-\Psi^{-1}(t) J_{f}(\overline{\mathbf{x}})$. Introducing a time-dependent coordinate transformation $\mathbf{W}(t)=\left(I_{N-1} \otimes \Psi^{-1}(t)\right) \tilde{\mathbf{Z}}(t)$ to system (15) yields

$$
\dot{\mathbf{W}}(t)=-c\left(\tilde{L}_{\sigma} \otimes I_{n}\right) \mathbf{W}(t)
$$

According to the matrix theory, there must exist a permutation matrix $A \in \mathbb{R}^{n(N-1) \times n(N-1)}$ such that $A^{\mathrm{T}}\left(\tilde{L}_{\sigma} \otimes I_{n}\right) A=I_{n} \otimes \tilde{L}_{\sigma}$. We then introduce a coordinate transformation $\overline{\mathbf{W}}(t)=A^{\mathrm{T}} \mathbf{W}(t)$ to system (A.1) 
to obtain

$$
\dot{\overline{\mathbf{W}}}(t)=-c\left(I_{n} \otimes \tilde{L}_{\sigma}\right) \overline{\mathbf{W}}(t)
$$

Obviously, system (A.2) is the combination of $n$ independent associated systems (17). Following the previous arguments, the solution of system (15) can be expressed by $\tilde{\mathbf{Z}}(t)=\sqrt{N}\left(I_{N-1} \otimes \Psi(t)\right) A^{-\mathrm{T}}(\mathbf{v} \otimes \mathbf{w}(t))$. Correspondingly, we obtain

$$
\|\tilde{\mathbf{Z}}(t)\| \leq \sqrt{N}\|\Psi(t)\| \cdot\|\mathbf{w}(t)\|
$$

The following provides how to estimate the bound of $\|\mathbf{w}(t)\|$ and $\|\Psi(t)\|$. Denote the convergence rate of system (17) as $\mu$, which is defined as

$$
\mu=-\sup _{\mathbf{w}\left(t_{0}\right) \neq 0} \lim _{t \rightarrow \infty} \frac{1}{t} \ln \frac{\|\mathbf{w}(t)\|}{\left\|\mathbf{w}\left(t_{0}\right)\right\|} .
$$

It is easy to verify from [Adrianova, 1995] that the equation $\mu \neq \pm \infty$ holds if and only if, for any $\epsilon>0$, the following conditions hold simultaneously

$$
\begin{gathered}
\lim _{t \rightarrow \infty} e^{(\mu+\epsilon) t}|| \mathbf{w}(t) \|=0, \\
\lim _{t \rightarrow \infty} e^{(\mu-\epsilon) t}\|\mathbf{w}(t)\|=\infty
\end{gathered}
$$

Apparently, if $\mu>0, \lim _{t \rightarrow \infty}\|\mathbf{w}(t)\|=0$, which further implies that $\lim _{t \rightarrow \infty} \| \mathbf{W}\left(t\left\|=\lim _{t \rightarrow \infty}\right\| \mathbf{Y}(t \|=0\right.$. Thus, the synchronization will be achieved. And if $\mu \leq 0$ for any bounded initial state $\mathbf{w}(0)$, synchronization cannot be achieved for system (17). By the definition of convergence rate $\mu$ and Eqs. (A.5)-(A.6), there exists a positive constant $k_{1}$, dependent on $\epsilon_{1}>0$, such that

$$
\|\mathbf{w}(t)\| \leq k_{1} e^{\left(-\mu+\epsilon_{1}\right)\left(t-t_{0}\right)}\left\|\mathbf{w}\left(t_{0}\right)\right\|
$$

for any positive scalar $\epsilon_{1}$.

And $\|\Psi(t)\|$ can be evaluated by the the largest Lyapunov exponent $\nu_{\max }$ of system (16) satisfying

$$
\|\Psi(t)\| \leq k_{2} e^{\left(\nu_{\max }+\epsilon_{2}\right)\left(t-t_{0}\right)}
$$

where $\epsilon_{2}$ is an arbitrary positive scalar and $k_{2}$ is a positive constant, dependent on $\epsilon_{2}$.

Therefore, it follows from Eqs. (A.3)-(A.8) that

$$
\|\tilde{\mathbf{Z}}(t)\| \leq k e^{\left(-\mu+\nu_{\max }+\epsilon\right)\left(t-t_{0}\right)}\left\|\tilde{\mathbf{Z}}\left(t_{0}\right)\right\|,
$$

where $\epsilon$ is an arbitrary positive scalar and $k$ is a positive constant, dependent on $\epsilon$. There is no doubt that one can select a sufficiently small $\epsilon$ such that $k \leq 1$. The proof is thus completed. 